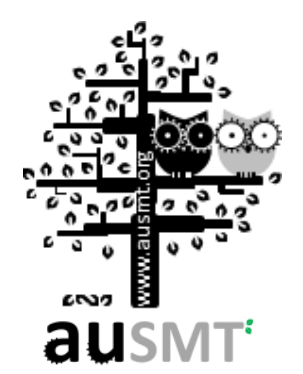

\title{
Uniform Design and Regression Analysis Methods for Optimal Operational Parameter Design of High-pressure Waterjet Machine
}

\author{
Sheng-Wen Huang ${ }^{1}$, Jyh-Horng Chou ${ }^{1,2}$, and Jinn-Tsong Tsai ${ }^{3, *}$ \\ ${ }^{1}$ Department of Electrical Engineering, National Kaohsiung University of Science and Technology, Kaohsiung, Taiwan \\ ${ }^{2}$ Department of Healthcare Administration and Medical Informatics, Kaohsiung Medical University, Kaohsiung, Taiwan \\ ${ }^{3}$ Department of Computer Science, National Pingtung University, Pingtung, Taiwan \\ (Received 7 December 2017; Accepted 26 December 2017; Published on line 1 June 2018) \\ *Corresponding author: jttsai@mail.nptu.edu.tw \\ DOI: 10.5875 /ausmt.v8i2.1675
}

Abstract: Currently, removing scales from harvested fish is a time- and labor-intensive process. To reduce labor costs and improve productivity, this study uses a uniform design and regression analysis methods to design optimal operational parameters of a high-pressure waterjet machine for removing tilapia scales. A uniform layout of $U_{10}{ }^{*}\left(10^{3}\right)$ was used to arrange three operational parameters for experiments. The removal rate is the quality index defined by the fish scale removal area divided by full surface area covered by scales. Experimental results show a removal rate of 85.39\%.

Keywords: fish scales; tilapia scales; uniform design method; regression analysis

\section{Introduction}

Removing fish scales is typically a time- and labor-intensive manual task. Attempts have been made to automate industrial scale fish descaling through machine-driven scraping, brushing and spraying with high-pressure waterjets. Such systems have been tested on fish of various weights, with results computed in terms of total area cleaned [1-5].

The present study designs a high-pressure waterjet machine (Fig. 1) to descale tilapia weighing 1 to 3 catties. Three operational parameters were found to influence the removal rate of fish scales, and the uniform design method [6] was used to identify the best combination of these three parameters. After each experiment, both sides of the cleaned fish were photographed, and the total cleaned and uncleaned areas were measured to calculate the removal rate. Regression analysis was used to build a regression model and identify the best operational parameter combination. Finally, the verified experiments were performed by using the operational parameter combination obtained by the regression model.

\section{Uniform Design Approach}

Wang and Fang [6] proposed a uniform design approach that can effectively deal with multiple level experiments, where the number of experiments is equal to the number of levels. In addition, the uniform design approach requires fewer experiments than the Taguchi method, thus reducing development costs [7].

Table 1 shows the uniform layout of $U_{10}{ }^{*}\left(10^{3}\right)$. The first column is the number of experiments and the other three columns are used for arranging three operational parameters of the high-pressure waterjet machine for experiments.

The steps of uniform design experiments are as follows:

Step 1: Clearly identify system problems.

Step 2: Identify the operational parameters that can affect the system.

Step 3: Select the range of parameters according to expert experience. 
Step 4: Choose an adequate uniform layout, according to the number of operational parameters and levels.

Step 5: Conduct experiments according to the uniform layout.

Step 6: Analyze the results using the image processing method and regression analysis. The optimal operational parameter values are obtained by the regression model.

Step 7: Conduct verification experiments to compare the results obtained by the practical experiments and the regression model.

Table 1. Uniform layout $U_{10}{ }^{*}\left(10^{3}\right)$.

\begin{tabular}{cccc}
\hline Exp. no. & $\mathbf{1}$ & $\mathbf{2}$ & $\mathbf{3}$ \\
\hline 1 & 1 & 5 & 7 \\
2 & 2 & 10 & 3 \\
3 & 3 & 4 & 10 \\
4 & 4 & 9 & 6 \\
5 & 5 & 3 & 2 \\
6 & 6 & 8 & 9 \\
7 & 7 & 2 & 5 \\
8 & 8 & 7 & 1 \\
9 & 9 & 1 & 8 \\
10 & 10 & 6 & 4
\end{tabular}

\section{Regression Analysis Approach}

Regression analysis [8] is used to investigate the strength of correlation between two or more variables. The following introduces unary and multiple linear regressions. Unary linear regression is a simple model used to reflect the relation between a variable and an output. The following Eq. (1) is a unary linear regression model.

$$
Y=\alpha+\beta X+\varepsilon
$$

In Eq. (1), where $Y$ is the dependent variable, $X$ is the independent variable, $\alpha$ and $\beta$ are regression parameters and $\varepsilon$ is a random error. Equation (1) denotes that the relations between $Y, X, \alpha, \beta$, and $\varepsilon$ are usually unknown values. This study has three operational parameters. Therefore, the relation should be assessed using multiple linear regression as in Eq. (2).

$$
Y=\alpha+\beta_{1} X_{1}+\beta_{2} X_{2}+\beta_{3} X_{3}+\varepsilon
$$

The Eq. (2) is similar to Eq. (1), but the number of independent variable $X$ and regression parameters $B$ are increased.

\section{Experimental Steps and Results}

\section{A. Experimental steps}

The experimental steps are described as follows.

Step 1: Use the uniform layout of $U_{10}{ }^{*}\left(10^{3}\right)$ for the three operational parameters of the high-pressure waterjet machine and their respective resolutions (see Table 1).

Step 2: Conduct experiments according to the uniform layout of $U_{10}{ }^{*}\left(10^{3}\right)$ and photograph the fish on both sides.

Step 3: Analyze the photographs using image processing software and calculate the removal rate for each experiment.

Step 4: Build the regression model and use it to find the best combination of three operational parameters and the removal rate of fish scales.

Step 5: Predict the maximal removal rate $Y$ and compare against the removal rate obtained by practice experiments.

Eq. (3) was used to compute the removal rate of fish scales.

$$
\text { Removal rate }=\frac{\text { Area of removed fish scales }}{\text { Area of full fish scales }} \times 100 \%
$$

Figure 1 shows the high-pressure waterjet machine to remove tilapia scales in the experiments. The red outline in Figure 2(a) shows the area of a tilapia originally covered by scales. The red outline in Figure 2(b) shows the area of the fish cleaned of scales. The cleaned area is nearly white and the color difference is used to assess the proportion of scales removed through image processing methods.

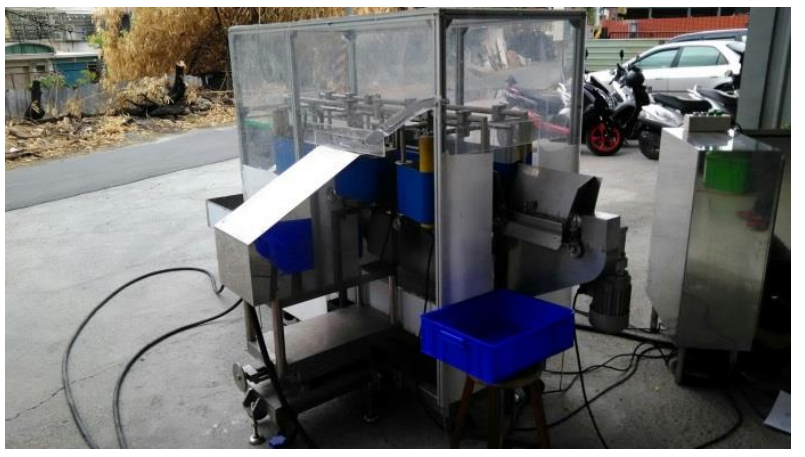

Figure 1. High-pressure waterjet machine used to remove tilapia scales.

B. Experimental results

Table 2 shows the operational parameter combinations and their removal rate results, where $x_{1}$ is 
the frequency for the speed of the machine chain $(27 \mathrm{~Hz}$ to $59.4 \mathrm{~Hz}), x_{2}$ is the water pressure $\left(17.5 \mathrm{~kg} / \mathrm{cm}^{2}\right.$ to $\left.26.95 \mathrm{~kg} / \mathrm{cm}^{2}\right), x_{3}$ is the frequency for the speed of the nozzle $(54.7 \mathrm{~Hz}$ to $79.9 \mathrm{~Hz})$. Each operational parameter combination was tested three times. The removal rate was obtained by averaging the results from the three experimental runs. Table 2 shows that the maximal removal rate $(84.47 \%)$ is obtained by the 4 th operational parameter combination in $U_{10}{ }^{*}\left(10^{3}\right)$.

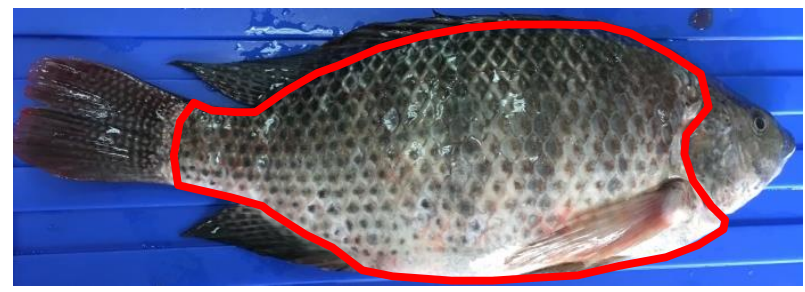

(a)

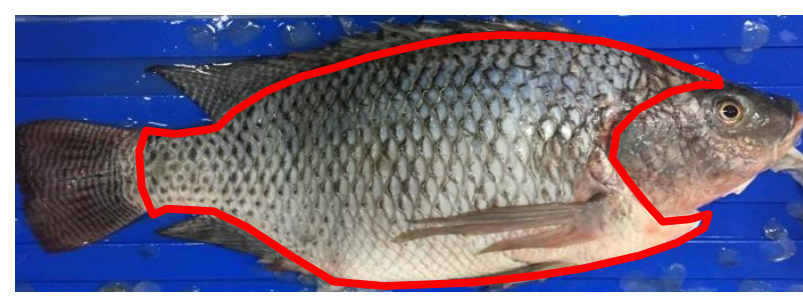

(b)

Figure 2. (a) Tilapia before cleaning and scale coverage (b) Tilapia after cleaning and scale removal coverage.

\section{Regression model}

The regression equation was generated by using the data of the $U_{10}{ }^{*}\left(10^{3}\right)$ and is shown below.

$$
y=-0.1294-0.0115 x_{1}+0.0443 x_{2}+0.0021 x_{3}
$$

where $y$ is the removal rate. The regression Eq. (4) shows that the items $x_{2}$ and $x_{3}$ are the more significant factors, because they have positive coefficients to obtain the maximal removal rate. The best operational parameters, obtained using the regression Eq. (4), are $x_{1}=27, x_{2}=$ 26.95 , and $x_{3}=79.9$, producing a removal rate of $92.18 \%$.

\section{Verification experiment}

The best operational parameters of $x_{1}=27, x_{2}=$ 26.95, and $x_{3}=79.9$, obtained using regression Eq. (4), were used to clean three different tilapia with the high-pressure waterjet machine, and achieved an average removal rate of $85.39 \%$.

\section{E. Discussion}

The operational parameter combination obtained by regression Eq. (4) produced a theoretical scale removal rate of $92.18 \%$, while the actual removal rate in verification experiments was $85.39 \%$, indicating a high degree of predictive accuracy. In addition, the scaling by machine costs about one-third that for manual scaling.

\begin{tabular}{ccccc}
\multicolumn{6}{c}{ Table 2. Results of the removal rate. } \\
\hline $\begin{array}{c}\text { Exp. } \\
\text { no. }\end{array}$ & $\boldsymbol{x}_{\mathbf{1}}$ & $\boldsymbol{x}_{\mathbf{2}}$ & $\boldsymbol{x}_{\mathbf{3}}$ & Removal rate (\%) \\
\hline 1 & 27 & 21.7 & 71.5 & 79.8244 \\
2 & 30.6 & 26.95 & 60.3 & 82.6307 \\
3 & 34.2 & 20.65 & 79.9 & 41.5756 \\
4 & 37.8 & 25.9 & 68.7 & 84.8752 \\
5 & 41.4 & 19.6 & 57.5 & 31.5651 \\
6 & 45 & 24.85 & 77.1 & 53.0873 \\
7 & 48.6 & 18.55 & 65.9 & 32.0307 \\
8 & 52.2 & 23.8 & 54.7 & 33.4255 \\
9 & 55.8 & 17.5 & 74.3 & 20.273 \\
10 & 59.4 & 22.75 & 63.1 & 41.709 \\
\hline
\end{tabular}

\section{Conclusions}

This study integrates the uniform design method and regression analysis to systematically identify operational parameters for a high-pressure waterjet machine used in removing tilapia scales. Experimental data obtained from the uniform layout of $U_{10} 0^{*}\left(10^{3}\right)$ were used to build a regression model for predicting the removal rate of tilapia scales. The regression model was also used to determine the optimal operational parameter combination for high-pressure waterjet scale removal. The maximal removal rate of tilapia scales obtained by the verification experiment is $85.39 \%$. The proposed approach can be used to optimize the automation of fish de-scaling, thus significantly reducing production costs and enhancing productivity.

\section{Acknowledgments}

This work was supported in part by the Ministry of Science and Technology, Taiwan, R.O.C., under grants MOST 106-2221-E-153-005 and MOST 105-2221-E-151-024-MY3. This work was supported in part by the Headquarters of University Advancement and Intelligent Manufacturing Research Center (iMRC), National Cheng Kung University, which are sponsored by the Ministry of Education, Taiwan, R.O.C. The authors thank Mr. Chih-Hsiung Chiou for his assistance in implementing the experiments. Financial support from the MIRDC is also gratefully acknowledged. 


\section{References}

[1] Z.-M. Ruan, M.-Y. Li, J.-Q. Peng, and Z.-M. Chen, "Improve and experiment the removing fish scales machine for milkfish," Journal of Agriculture and Forestry, vol. 53, no. 4, pp. 43-44, 2013.

[2] Y.-A. Chan, "Design of Grouper Scaling Machine," M. S. thesis, Department of Bio-Industrial Mechatronics Engineering, College of Bioresources and Agriculture, National Taiwan University, 2016.

[3] Y.-C. Lee, C.-H. Chiou, J.-C. Tseng, and S.-E. Tsai, "Design of grouper automatic processing machine," Research Report 104AS-3.4.2-FA-F2, Fisheries Agency, Council of Agriculture, Executive Yuan, Taiwan, 2015.

[4] J.-J. Wang, L. Zong, and S.-B. Xiong, "Continuous scaling method for freshwater fish," Transactions of the Chinese Society of Agricultural Engineering, vol. 27, no. 9, pp. 339-343, 2011.
[5] J.-J. Wang, L. Zong, and S.-B. Xiong, "Study on scale bio-adhesion force and scaling properties for freshwater fish," Transactions of the Chinese Society of Agricultural Engineering, vol. 28, no. 3, pp. 288-292, 2012.

[6] Y. Wang and K.-T. Fang, "A Note on Uniform Distribution and Experimental Design," Kexue Tongbao, vol. 26, no. 6, pp. 485-489, 1981.

[7] J.-H. Chou, "Optimization Approaches Lecture Notes and Handouts," the Institute of Electrical Engineering, National Kaohsiung University of Applied Sciences, Taiwan, 2016.

[8] M. A. Efroymson, "Multiple regression analysis," In Ralston A. and Wilf, H. S. (eds.), Mathematical Methods for Digital Computers, New York: Wiley, 1960. 